\title{
Qualidade de Vida em Pacientes Submetidos à Cirurgia Ortognática: Saúde Bucal e Autoestima
}

Quality of Life in Patients Who Undergo Orthognathic Surgery: Oral Health and Self-Esteem

Calidad de Vida en Pacientes Sometidos Cirugía Ortognática: Salud Bucal y Autoestima

Rubens Guimarães Filho, Eurico Candido Oliveira Junior, Thammy Rebeca Mesquita Gomes \& Tiago Dimas Antunes de Souza Universidade de Taubaté 
Resumo: A cirurgia ortognática é o ramo da cirurgia bucomaxilofacial que trata das deformidades dentofaciais, buscando a correção da oclusão e melhora articular, respiratória e fonética, que geram benefícios funcionais e harmonizam a face, o que acarreta aspectos positivos em relação à estética e ao fator psicossocial do paciente. Objetivou-se avaliar a qualidade de vida desses pacientes, através dos aspectos funcionais, estéticos e psicossociais. O presente estudo foi realizado em clínica odontológica particular, na cidade de Taubaté. Foram avaliados 33 pacientes, 12 do gênero masculino e 21 do gênero feminino, com idades entre 18 a 45 anos, com tratamento ortocirúrgico indicado ou já realizado, utilizando-se os questionários Escala de Autoestima de Rosenberg/UNIFESP-EPM, Oral Health Impact Profile-14 - OHIP ${ }_{14}$ ePIDAQ-Brasil/UNIFESP e o Teste não Paramétrico de Mann-Whitney. Os resultados evidenciaram que a cirurgia ortognática é um procedimento capaz de oferecer melhora da qualidade de vida para pacientes portadores de deformidades dentofaciais, por meio dos aspectos funcionais, estéticos e psicossociais.

Palavras-chave: Cirurgia ortognática. Qualidade de vida. Anormalidades maxilofaciais. Autoestima. Saúde bucal.

Abstract: The orthognathic surgery is the branch of oral and maxillofacial surgery that deals with the dentofacial deformities, seeking correction of the occlusion, and joint, respiratory and speech improvement, generating functional benefits and harmonizing the face, which leads to positive aspects of aesthetics and psychosocial factors of the patient. The objective was to evaluate the quality of life of patients through the functional, aesthetic and psychological aspects. This study was conducted in a private dental practice in Taubaté. We evaluated 33 patients, 12 males and 21 females, aged 18-45 years with orthosurgical treatment indicated or already accomplished, using the questionnaires Scale Rosenberg Self-Esteem/ UNIFESP-EPM, Oral Health Impact Profile-14 - OHIP14 and PIDAQ-Brasil/UNIFESP and the Non-parametric Test of Mann Whitney. The results showed improved self-esteem, lower impact of oral health problems on individual health and showed no significant difference in the improvement of dental aesthetics. Orthognathic surgery is a procedure capable of providing improved quality of life for patients with dentofacial deformities by means of functional, aesthetic and psychosocial factors.

Keywords: Orthognatic surgery. Quality of life. Maxillofacial abnormalities. Self-esteem. Oral health.

Resumen: La cirugía ortognática es la rama de la cirugía oral y maxilofacial que el cuidado de las deformidades dentofaciales, buscando la corrección de la oclusión, mejora la respiración conjunta y el habla, la generación de beneficios funcionales y la armonización de la cara, lo que conduce a los aspectos positivos de la estética y los factores psicosociales de el paciente. El objetivo fue evaluar la calidad de vida de los pacientes por medio de los aspectos funcionales, estéticos y psicológicos. Este estudio se llevó a cabo en la práctica dental privada en la ciudad de Taubaté. Se evaluaron 33 pacientes, 12 hombres y 21 mujeres, con edades entre 18-45 años con el tratamiento indicado ortocirúrgico o ya logrado, utilizando cuestionarios Escala de Autoestima de Rosenberg/UNIFESP-EPM, Oral Health Impact Profile-14 - OHIP14 y PIDAQ-Brasil/UNIFESP y la prueba non paramétrica Mann Whitney. Los resultados mostraron impacto mejorado la autoestima, la menor de los problemas de salud bucal en la salud individual y no mostró ninguna diferencia significativa en la mejora de la estética dental. La cirugía ortognática es un procedimiento capaz de proporcionar una mejor calidad de vida para los pacientes con deformidades dentofaciales mediante factores funcionales, estéticos y psicosociales.

Palabras-cavle: Ortognática cirugía. Calidad de vida. Anomalías maxilofaciales. Autoestima. Salud oral.

A cirurgia ortognática trata das deformidades dentofaciais, possibilitando a correção da oclusão inadequada, melhora da condição respiratória, elevação da autoestima, maior satisfação com a estética facial e dental e melhora ou resolução de possíveis dores musculares e/ou articulares. Esse tratamento, contudo, não está isento de falhas, e é importante adequada indicação, planejamento, preparação psicológica prévia do paciente e da família, preparo pré-operatório do caso, transcirúrgico sem intercorrências e cuidados pós-operatórios.
A cirurgia ortognática é o ramo da cirurgia bucomaxilofacial que se preocupa com as correções das deformidades dentofaciais, sendo esses casos tratados por uma equipe multidisciplinar, coordenada pelo cirurgião bucomaxilofacial e pelo ortodontista. A conduta de tratamento, nesses casos, visa a atender à harmonia facial e à dentária, à oclusão funcional, à saúde das estruturas orofaciais e à estabilidade do procedimento (Laureano, Carvalho, Gomes, Bessa, \& Camargo, 2002). 
As técnicas cirúrgicas ortognáticas e seu impacto

psicossocial

comprovam os

benefícios obtidos

com o resultado

da sua realização, incluindo

aumento da

autoestima e melhor convívio social

(Hunt, Johnston \& Hepper, 2001).
Laureano, Silva, Vasconcellos, Silva e Rocha (2005) afirmam que a cirurgia ortognática consiste no procedimento de escolha para o tratamento das deformidades dentoesqueléticas severas, que visam à correção da deficiência funcional e que acarretam modificações estéticas no paciente.

Problemas graves de maloclusão que requerem um tratamento combinado de ortodontia e de cirurgia ortognática são denominados deformidades dentofaciais, para diferenciar daqueles menos severos que podem ser tratados somente com ortodontia. Quem necessita de tratamento cirúrgico em adição à ortodontia são aqueles portadores de problemas esqueletais e/ou dentoalveolares cuja magnitude é excessiva para a resolução apenas com ortodontia (Araújo, 1999).

Lima, Moro, Tanaka, Fattah e Renon (1999) citam as condições que podem indicar a cirurgia ortognática, estando entre elas dificuldade na mastigação, problemas de fala, dor maxilomandibular crônica, apinhamentos dentários excessivos, dificuldade de abertura bucal, mordida aberta, falta de balanceamento da aparência facial, injúrias faciais ou defeitos congênitos, mento retraído, mandíbula protruída, incompetência labial, respiração bucal crônica e apneia do sono.

Para Ribas, Reis, França e Lima (2005), é importante conhecer as expectativas dos pacientes, pois estas estão relacionadas diretamente com o grau de satisfação pós-operatório. Fatores como pessimismo, ansiedade e pacientes com pobre suporte social apresentam maiores riscos de complicações. Quanto menos ansioso for o paciente, maior será a chance de sucesso e de satisfação.

A cirurgia ortognática trata da correção cirúrgica das deformidades dentofaciais, e a sua importância está não só na correção da oclusão mas também da estética facial. Isso significa que os aspectos psicossociais estão diretamente relacionados a esse tipo de tratamento, pois a aparência facial influencia a formação da imagem corporal, da identidade e da autoestima (Nicodemo, Pereira \& Ferreira, 2007).

A relação maxilomandibular corrigida pela cirurgia ortognática favorecerá a função mastigatória, a fonética, a respiração e a estética facial, portanto, muitas implicações estão envolvidas nesse tratamento cirúrgico, pois as mudanças faciais repercutem na vida pessoal e social do indivíduo, e, por vezes, o componente psicológico do paciente deverá ser preparado para receber um procedimento cirúrgico de tal magnitude (Ribas et al., 2005).

As técnicas cirúrgicas ortognáticas e seu impacto psicossocial comprovam os benefícios obtidos com o resultado da sua realização, incluindo aumento da autoestima e melhor convívio social (Hunt, Johnston \& Hepper, 2001).

Instrumentos para avaliação da qualidade de vida relacionada à saúde bucal têm sido desenvolvidos, e, em sua maioria, destinamse a comparar resultados de tratamentos no contexto de estudos clínicos (Castro, Portela \& Leão, 2007).

É de extrema importância avaliar a percepção do indivíduo sobre as alterações geradas pelo tratamento cirúrgico em relação a seus padrões, desejos e preocupações, pois, como já verificado em estudo anterior por Cunningham, Garrat e Hunt (2002), a percepção do indivíduo acerca de si mesmo, do seu estado físico e emocional é indispensável para direcionar o tratamento, e demonstram a inter-relação entre saúde bucal, saúde geral e qualidade de vida.

Qualidade de vida refere-se à percepção do indivíduo de sua posição na vida, no contexto da cultura e no sistema de valores no qual ele vive e em relação aos objetivos, expectativas, padrões e preocupações (The Whoqol Group, 1998). Para Seidl e Zannon (2004), a qualidade de vida tem grande importância no cotidiano, pois pode contribuir para aumentar a integralidade da assistência na perspectiva da saúde como direito de cidadania. 
De acordo com a definição de qualidade de vida relacionada à saúde bucal, apenas a ausência de doenças e de disfunções bucofaciais não são consideradas suficientes para indicação de boa saúde bucal (Klages, Buckner, \& Zentner, 2004). Esta está intimamente ligada à saúde geral, por isso a necessidade da mensuração da qualidade de vida em pacientes submetidos à cirurgia ortognática, avaliando o impacto que a deformidade dentofacial pode gerar à sua saúde física, mental e social, e o interesse em compreender as melhoras funcionais, psicossociais e estéticas obtidas através da cirurgia ortognática.

Diante do exposto, o objetivo deste trabalho foi avaliar a qualidade de vida de pacientes submetidos à cirurgia ortognática, através dos aspectos funcionais, estéticos e psicossociais, por meio de questionários cientificamente validados.

\section{Materiais e métodos}

O estudo foi aprovado pelo Comitê de Ética e Pesquisa sob o Protocolo no 125/2010, de acordo com a Resolução no 196/96, do Ministério da Saúde e suas complementações.

A casuística foi constituída por pacientes escolhidos consecutivamente em clínica odontológica particular, na cidade de Taubaté-SP.

Foram avaliados 35 pacientes, 14 do sexo masculino e 21 do sexo feminino, com idades entre 18 a 45 anos, com tratamento ortocirúrgico indicado ou já realizado.

Todos os pacientes foram esclarecidos pelo autor sobre a pesquisa. Antes de participarem, o Termo de Consentimento Livre e Esclarecido e o Termo de Consentimento da Participação da Pessoa como sujeito foram devidamente lidos e explicados. Os termos foram dados ao paciente, que os assinou e datou, sendo mantida uma cópia com o entrevistador e oferecida uma cópia ao entrevistado.
Para seleção dos pacientes, foram considerados os respectivos critérios de inclusão e exclusão: indivíduos de 18 a 65 anos de idade, sem restrição quanto a sexo, religião, raça, etnia ou escolaridade, e indivíduos menores de 18 e maiores de 65 anos de idade, com doença sistêmica não controlada, com desordens sanguíneas hereditárias ou adquiridas, com problemas psiquiátricos e cirurgias faciais prévias.

Os pacientes foram avaliados em dois períodos, pré e pós-operatório. O préoperatório refere-se ao período em que se submeteram ao preparo ortodôntico, não sendo determinado previamente um período de tempo mínimo e máximo de tratamento, e o pós-operatório, depois de passado ao menos um mês da intervenção cirúrgica, que consistiu de avanço ou no recuo da maxila, retrusão discreta ou protusão mandibular, segmentação e impactação da maxila e mentoplastia, não sendo necessariamente efetuadas todas essas correções em um mesmo paciente e existindo em alguns casos combinação de movimentos dos maxilares.

A avaliação foi realizada com questionários validados cientificamente e aplicados pelo pesquisador na forma de entrevistas.

Todos os indivíduos que concordaram em participar da pesquisa preencheram três questionários.

O Questionário de Impacto Psicossocial da Estética Dental (PIDAQ-Brasil/UNIFESP) (Vieira, 2010) é um instrumento específico para avaliação de qualidade de vida relacionada à saúde bucal em adultos jovens. É constituído por 23 itens que avaliam quatro dimensões da qualidade de vida relacionada à saúde bucal: autoconfiança dental, impacto social, impacto psicológico e aspectos estéticos, sendo que cada uma delas é avaliada separadamente. Também, ao final, o instrumento gera um valor total e pode ser avaliado como um todo. Para avaliação dos resultados, são dados escores para cada questão em uma escala do tipo Likert, valores de zero a quatro. Os valores 
totais finais ficam entre zero e 92. O menor escore indica a melhor qualidade de vida, tanto para as dimensões quanto para o instrumento todo.

Já a Escala de Autoestima de Rosenberg/UNIFESP-EPM é um questionário específico para a avaliação da autoestima, composto por dez questões. Para avaliação dos resultados, é atribuído um escore a cada resposta. Esses escores são somados e obtém-se um único escore total, que é avaliado. A pontuação do questionário varia de zero a trinta, sendo zero o melhor estado de autoestima e trinta, o pior estado (Dini, Quaresma \& Ferreira, 1991).

O questionário Oral Health Impact Profile-14 - $\mathrm{OHIP}_{14}$ avalia como alterações nos dentes ou na boca podem causar problemas na vida diária dos indivíduos. Para a análise do $\mathrm{OHIP}_{14^{\prime}}$ as respostas ordinais foram graduadas como 0 para nunca e até quatro para sempre, e todas as respostas ordinais foram somadas para produzir um escore total do $\mathrm{OHIP}_{14^{\prime}}$ que poderia variar de zero a 56, com maiores escores significando impacto mais negativo na saúde oral (DeOliveira \& Nadanovsky, 2005).

Para análise estatística dos resultados, foi utilizado teste não paramétrico. Aplicou-se o Teste de Mann-Whitney para comparar os escores obtidos. Para exibição gráfica da amostra, foi utilizado o aplicativo Microsoft Office Excel 2003.

\section{Resultados}

Foram entrevistados 18 pacientes que não haviam se submetido à cirurgia ortognática, e a pontuação obtida em cada questionário respondido foi somada e exibida na Tabela 1. Esses valores exibidos foram os utilizados para avaliação dos resultados.

Tabela 1. Resultados dos testes Escala de Autoestima de Rosenberg/UNIFESP-EPM, OHIP14 e PIDAQ nos pacientes não operados

\begin{tabular}{llll}
\hline Paciente & Rosenberg & OHIP14 & PIDAQ \\
\hline 1 & 12 & 59 & 71 \\
2 & 10 & 29 & 16 \\
3 & 11 & 30 & 15 \\
4 & 2 & 14 & 16 \\
5 & 9 & 43 & 36 \\
6 & 11 & 30 & 57 \\
7 & 3 & 41 & 43 \\
8 & 15 & 56 & 31 \\
9 & 10 & 49 & 46 \\
10 & 12 & 38 & 53 \\
11 & 13 & 32 & 28 \\
12 & 12 & 54 & 31 \\
13 & 3 & 62 & 44 \\
14 & 10 & 16 & 17 \\
15 & 12 & 30 & 30 \\
16 & 9 & 22 & 23 \\
17 & 1 & 31 & 21 \\
18 & 12 & 47 & 33 \\
19 & - & - & - \\
20 & - & - & - \\
\hline
\end{tabular}


Foram entrevistados 17 pacientes que haviam se submetido à cirurgia ortognática, e a pontuação obtida em cada questionário respondido foi somada e exibida na Tabela 2. Esses valores exibidos foram os utilizados para avaliação dos resultados.

Tabela 2. Resultados dos testes Escala de Autoestima de Rosenberg/UNIFESP-EPM, OHIP14 e PIDAQ nos pacientes operados

\begin{tabular}{llll}
\hline Paciente & Rosenberg & OHIP ${ }^{14}$ & PIDAQ \\
\hline & & & \\
1 & 4 & 16 & 38 \\
2 & 10 & 39 & 25 \\
3 & 4 & 18 & 24 \\
4 & 7 & 14 & 21 \\
5 & 10 & 14 & 17 \\
6 & 9 & 23 & 28 \\
7 & 6 & 20 & 21 \\
8 & 2 & 29 & 20 \\
9 & 6 & 25 & 30 \\
10 & 11 & 31 & 28 \\
11 & 5 & 24 & 26 \\
12 & 8 & 28 & 23 \\
13 & 0 & 16 & 25 \\
14 & 6 & 28 & 23 \\
15 & 7 & 48 & 23 \\
16 & 8 & 44 & 24 \\
17 & 12 & 40 & 22 \\
18 & - & - & - \\
19 & - & - & - \\
20 & - & - & - \\
& - & & \\
\hline
\end{tabular}

Para avaliação dos resultados, foi utilizado o Teste de Mann-Whitney, que é um teste não paramétrico usado para avaliação da significância de duas amostras independentes.

Fixou-se em 0,05 ou 5\% o nível de rejeição da hipótese de nulidade, assinalando-se com um asterisco $(*)$ os valores estatisticamente significantes.

Rosenberg

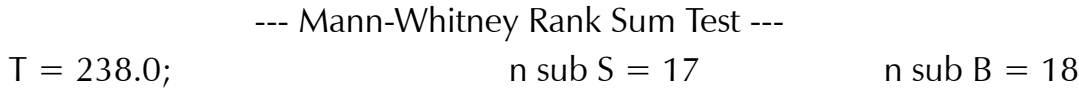

O tamanho da amostra é grande o suficiente para usar a aproximação normal a fim de testar a significância de T.

$\mathrm{Z} \operatorname{sub} \mathrm{T}=2.239 \quad \mathrm{P}=0.025^{*}$

Esse resultado indicou que $\mathrm{P}$ foi estatisticamente significante, ou seja, que há uma diferença entre os dois grupos e que existe apenas 2,5\% de chance de errar ao dizer que os pacientes operados têm melhor autoestima que os não operados, portanto, que a autoestima dos operados é melhor. 


$$
\mathrm{OHIP}_{14}
$$

--- Mann-Whitney Rank Sum Test ---

$\mathrm{T}=232.0 ; \quad \mathrm{n}$ sub $\mathrm{S}=17 \quad \mathrm{n}$ sub $\mathrm{B}=18$

O tamanho da amostra é grande o suficiente para usar a aproximação normal a fim testar a significância de T.

Z sub $\mathrm{T}=2.428 \quad \mathrm{P}=0.015^{*}$

Esse resultado indicou que $P$ foi estatisticamente significante, ou seja, que há uma diferença entre os dois grupos, e que existe apenas 1,5\% de chance de errar ao dizer que os pacientes operados têm menor impacto com os problemas bucais que os não operados. Os operados, portanto, têm menores problemas bucais, ou, pelo menos, estes não geram impacto em sua qualidade de vida.

\section{PIDAQ}

--- Mann-Whitney Rank Sum Test ---

$\mathrm{T}=253.5 ; \quad \mathrm{n}$ sub $\mathrm{S}=17 \quad \mathrm{n}$ sub $\mathrm{B}=18$

O tamanho da amostra é grande o suficiente para usar a aproximação normal a fim de testar a significância de T.

$Z$ sub $T=1.719 \quad P=0.086$

Esse resultado indicou que $\mathrm{P}$ foi estatisticamente significante, ou seja, que não há diferença suficiente entre os dois grupos, e que existe $8,6 \%$ de chance de errar ao dizer que os pacientes operados têm melhor estética dental que os não operados. Para que possa ser considerada verdade, $\mathrm{P}$ tem que ser igual ou menor que 0,05 , o que não foi o caso, portanto, os operados e os não operados têm a mesma estética dental.

\section{Discussão}

A maioria dos pacientes que participaram deste estudo tinha idade acima dos 25 anos $(56,8 \%)$, discordando da literatura que observa que os pacientes submetidos à cirurgia ortognática têm buscado esse tratamento quando mais jovens, porém é semelhante ao estudo de Aghabeigi, Hiranaka, Keith e Kelly (2001), que relata a média de idade dos pacientes em 28,5 anos. Nas amostras obtidas por Nicodemo, Pereira e Ferreira (2008), a maioria dos pacientes possuía entre 17 a 22 anos (86\%). Em nosso estudo, a amostra, em sua maior parte, é composta pelo sexo feminino (60\%), como encontrado por Nicodemo et al. (2007), que possuía, em sua amostra, a maior parte composta pelo sexo feminino (55\%), e Westermarck, Shayeghi e Thor (2001), com um valor de $63 \%$ para o sexo feminino.

Não foram encontrados estudos na literatura nacional que exibissem dados comparativos em relação ao nível de escolaridade equivalente ao apresentado neste estudo, nem dados comparativos para estudos internacionais, já que o nível de escolaridade em outros países possui uma distribuição diferente do padrão nacional.

O interesse pelo tema qualidade de vida tem crescido nas práticas desenvolvidas na área da saúde, fomentado pesquisas científicas e tem sido cada vez mais buscado pelas pessoas em seu cotidiano, o que contribui para melhor saúde física, mental e social. Os autores Klages et al. (2004), Seidl e Zannon, e The Whoqol Group $(1995,1998)$ denominam qualidade de vida ao entendimento que o indivíduo possui sobre si mesmo, à posição que ocupa na sociedade e no seu sistema de valores e à maneira que relaciona esses fatores às suas expectativas, interesses e anseios, sabendo que qualidade de vida não está simplesmente em ter condições básicas de vida. 
Isso significa

que os aspectos

psicossociais

estão diretamente relacionados

a esse tipo de

tratamento, pois a aparência facial influencia a formação da imagem corporal, da identidade e da autoestima (Nicodemo et al., 2007).
Para a literatura pertinente pesquisada, a cirurgia ortognática consiste de um procedimento realizado em conjunto pelo ortodontista e pelo cirurgião bucomaxilofacial que trata da correção cirúrgica das deformidades dentofaciais que têm importância na correção da oclusão e da estética facial. Isso significa que os aspectos psicossociais estão diretamente relacionados a esse tipo de tratamento, pois a aparência facial influencia a formação da imagem corporal, da identidade e da autoestima (Nicodemo et al., 2007).

O resultado da cirurgia ortognática tem impacto direto na melhora da qualidade de vida dos pacientes, seja funcional, estética ou psicossocial, pois inúmeros são os benefícios que podem ser gerados por meio dela.

Um questionário sobre qualidade de vida (Derriford Scale - DAS59) revelou que 101 pacientes $(84,2 \%$ da amostra) reportaram mudanças positivas na qualidade de suas vidas após a cirurgia ortognática em um estudo conduzido por Sadek e Salem (2007). Já neste estudo, foram utilizados três questionários sobre qualidade de vida, tendo 35 pacientes relatado o mesmo fato.

Ribas et al (2005). ressaltam que a cirurgia ortognática é um procedimento que pode promover melhora significativa da qualidade de vida das pessoas portadoras de deformidades dentofaciais, melhorando a autoestima, a mastigação e a fonação, resultados semelhantes aos encontrados neste estudo.

Na presente pesquisa, as correções das deformidades faciais por meio da cirurgia ortognática consistiram de avanço ou recuo da maxila, retrusão discreta ou protusão mandibular, segmentação da maxila, impactação da maxila e mentoplastia, não tendo necessariamente sido efetuadas todas essas correções em um mesmo paciente e existindo, em alguns casos, combinação de movimentos dos maxilares. $\mathrm{O}$ reposicionamento anterior de mandíbula é um dos procedimentos mais comuns realizados na cirurgia ortognática, e normalmente está indicado para pacientes que apresentam oclusão classe II de Angle (Bell,1992). Segundo Turvey, Phillips, Zaytoun e Proffit (1988), em certos casos, a discrepância apresentada pelo paciente não permite resolução apenas com mobilização de um segmento, sendo indicado um procedimento combinado. A mentoplastia é descrita por alguns autores como um refinamento da técnica, pelo ganho estético adicional na projeção do mento, no sentido ânteroposterior, latero-lateral e crânio-caudal (Araújo, 1999). A maioria dos casos conduzidos no estudo de Aghabeigi, Hiranaka, Keith e Kelly foi tratada com uma impacção maxilar Le Fort I isoladamente (46\%) ou em combinação com um processo mandibular bilateral como osteotomia intrabucal oblíqua (37\%) ou bilateral sagital (14\%).

Os resultados encontrados neste estudo indicaram que pacientes operados têm melhor autoestima que os não operados. Em estudo conduzido por Shaw (1981), e por Nicodemo, Pereira e Ferreira (2008), foram discutidos os aspectos sociais de uma deformidade dentofacial, e foi demonstrado que os pacientes não tratados possuíam baixa autoestima. Há concordância entre o resultado obtido por Gomes, Rapoport, Lehn, Denardim e Carlini (2008) e este trabalho, no qual foi verificado aumento da autoestima em pacientes com deformidade dentofacial após a realização da cirurgia. No estudo de Ribas et al. (2005,) foi constatada melhora nas relações interpessoais, na integração social e no aumento da autoestima, mais uma vez, o que se assemelha ao resultado obtido por este estudo. Em pesquisa conduzida por Motegi, Hatch, Rugh e Yamaguchi (2003), foi constatada melhora na integração social, aumento da autoestima e redução da 
ansiedade, da hostilidade e da depressão após a realização da cirurgia.

Os pacientes operados sofrem menor impacto dos problemas bucais que os não operados ou têm menores problemas bucais, que não geram impacto em sua qualidade de vida, e essa situação também foi encontrada em estudos realizados por Ribas et al., Nisalak, Suddhasthira e Katakul (2006), Modig, Andersson e Wardh (2006) e Murphy, Kearns, Sleeman, Cronin e Allen (2011). Foi relatado ainda melhora na mastigação e na fonação. Resultado funcional satisfatório também foi verificado na pesquisa de Motegi et al., na qual foi feita uma comparação entre pacientes pré e pós-operados, em que os pós-operados apontaram melhora da função bucal e do quadro de dor. Em estudo conduzido por Zhou, Hagg e Rabie (2001), foram constatadas melhoras funcionais com relação à melhora na mastigação e nas dores articulares.
A avaliação dos pacientes operados e dos não operados não exibiu diferenças significativas em relação à estética dental, diferentemente do relatado em estudos realizados por Nisalak et al. e Rustemeyer e Gregersen (2012), que concluíram ser a estética dental muito satisfatória para a maioria dos pacientes.

É interessante pesquisar com maior abrangência e lucidez a condição da melhoria da estética obtida ou não, já que esse conceito é de difícil avaliação e que cada indivíduo possui um padrão de beleza almejado para si e para avaliação do outro.

\section{Conclusão}

A cirurgia ortognática é um procedimento capaz de oferecer melhora da qualidade de vida para pacientes portadores de deformidades dentofaciais, por meio dos aspectos funcionais, estéticos e psicossociais.

\section{Rubens Guimarães Filho}

Doutor em Cirurgia e Traumatologia Bucomaxilofacial pela Universidade Estadual de Campinas e Professor Adjunto III da Disciplina de Cirurgia e Traumatologia Bucomaxilofacial da Universidade de Taubaté, Taubaté SP - Brasil.

E-mail: contato@institutoodontologico.com.br

\section{Eurico Candido Oliveira Junior}

Especializando em Cirurgia e Traumatologia Bucomaxilofacial na Universidade Veiga de Almeida, Rio de Janeiro $-\mathrm{RJ}$ - Brasil.

E-mail: euricobmf@gmail.com

\section{Thammy Rebeca Mesquita Gomes}

Graduada em Odontologia pela Universidade de Taubaté, Taubaté - SP - Brasil.

E-mail: thammyrebeca@hotmail.com

\section{Tiago Dimas Antunes de Souza}

Graduando em Psicologia na Universidade de Taubaté, Taubaté - SP - Brasil.

E-mail: tiagodimass@hotmail.com

\section{Endereço para envio de correspondência:}

Hospital Universitário de Taubaté - Setor Cirurgia e Traumatologia Bucomaxilofacial. Avenida Granadeiro Guimarães, 270, Centro. CEP: 12020-130. Taubaté, SP. 


\section{Referências}

Aghabeigi, B., Hiranaka, D., Keith, D. A., \& Kelly, J. P. (2001). Effect of orthognathic surgery on the temporomandibular joint in patients with anterior open bite. Int. J. Adult Orthod. Orthognath. Surg., 16(2), 153-160.

Araújo, A. (1999). Cirurgia ortognática. São Paulo: Livraria Editora Santos.

Bell, W. H. (1992). Modern practice in orthognathic and reconstructive surgery. Pennsylvania: W. B. Saunders.

Castro, R. A., Portela, M. C., \& Leão, A. T. (2007). Adaptação transcultural de índices de qualidade de vida relacionada à saúde bucal. Cad. Saúde Pública, 23(10), 2275-2284.doi: http://dx.doi.org/10.1590/S0102-311X2007001000003

Cunningham, S. J., Garrat, A. M., \& Hunt, N. P. (2002). Development of a condiction-specific quality of life measure for patients with dentofacial deformity : II. Validity and responsiveness testing. Community Dent. Oral Epidemiol., 30, 81-90. doi: 10.1034/j.1600-0528.2002.300201.x

DeOliveira, B. H., \& Nadanovsky, P. (2005). Psychometric properties of the Brazilian version of the Oral Health Impact Profile-short form. Community Dent. Oral Epidemiol., 33, 307-314. doi: 10.1111/j.1600-0528.2005.00225.x

Dini, G. M., Quaresma, M. R., \& Ferreira, L. M. (1991). Adaptação cultural e validação da versão brasileira da escala de autoestima de Rosenberg. Rev. Soc. Bras. Cir. Plast., 19(1), 47-52.

Gomes, K. U., Rapoport, A., Lehn, C. N., Denardim, O. V. P., \& Carlini, J. L. (2008). O impacto na qualidade de vida após o reposicionamento cirúrgico da pré-maxila em portadores de fissura lábio palatal bilateral - estudo de 50 casos. Rev. Col. Bras. Cir., 35(6), 361-367. doi: http://dx.doi.org/10.1590/ S0100-69912008000600004

Hunt, O. T., Johnston, C. D., \& Hepper, P. G. (2001). The psychosocial impact of orthognathic surgery: A systematic review. Am. J. Orthod. Dentofacial Orthop., 120, 490-497. doi:10.1067/mod.2001.118402

Klages, U., Buckner, A., \& Zentner, A. (2004). Dental aesthetics, self-awareness, and oral health related quality of life in young adults. Euro J. Ortho., 26(5), 507-514. doi: 10.1093/ ejo/26.5.507

Laureano, J. R. F., Carvalho, R., Gomes, A. C. A., Bessa, R. N., \& Camargo I. B. (2002). Cirurgia ortognática combinada: relato de um caso clínico. Rev. Cir. Traumat. Buco-MaxiloFacial, 1(2), 31-41.

Laureano, J. R. F., Silva, E. D. O., Vasconcellos, R. J. H., Silva, L. C. F., \& Rocha, N. F. (2005). Alterações em discrepâncias antero-posteriores na cirurgia ortognática. Rev. Cir. Traumatol. Buco-Maxilo-Fac., 5(1), 45-52.

Lima, N. J., Moro, M. A., Tanaka, F. Y., Fattah, C. M. R. S., \& Renon, M. A. (1999). O que significa cirurgia ortognática? Arq. Ciên. Saúde Unipar, 3(3), 273-276.

Modig, M., Andersson, L., \& Wardh, I. (2006). Patients' perception of improvement after orthognathic surgery: Pilot study. British Journal of Oral and Maxillofacial Surgery, 44, 24-27. doi: http://dx.doi.org/10.1016/j.bjoms.2005.07.016

Motegi, E., Hatch, J. P., Rugh, J. D., \& Yamaguchi, H. (2003). Health-related quality of life and psychosocial function 5 years after orthognathic surgery. Am. J. Orthod. Dentofacial Orthop., 124, 138-143. doi: http://dx.doi.org/10.1016/ S0889-5406(03)00391-3
Murphy, C., Kearns, G., Sleeman, D., Cronin, M., \& Allen, P. F. (2011). Clinical relevance of orthognathic surgery on quality of life. Int. J. Oral Maxillofac. Surg., 40(9), 926-930. doi: http://dx.doi.org/10.1016/j.ijom.2011.04.001

Nicodemo, D., Pereira, M. D., \& Ferreira, L. M. (2007). Cirurgia ortognática: abordagem psicossocial em pacientes classe III de angle submetidos à correção cirúrgica da deformidade dentofacial. Rev. Dental Press. Ortodon. Ortop. Facial, 12(5), 46-54. doi: http://dx.doi.org/10.1590/S141554192007000500007

Nicodemo, D., Pereira, M. D., \& Ferreira, L. M. (2008). Effect of orthognathic surgery for class III correction on quality of life as measured by SF-36. Int. J. Oral Maxillofac. Surg., 37(5), 131-134.

Nisalak, P., Suddhasthira, T., \& Katakul, B. (2006). Patient's perception and satisfaction following orthognathic surgery. Mahidol Dent. J., 26, 267-279.

Ribas, M. O., Reis, L. F. G., França, B. H. S., \& Lima, A. A. S. (2005). Cirurgia ortognática: orientações legais ortodontistas e cirurgiões bucofaciais. Rev. Dental Press. Ortodon. Ortop. Facial, 10(6), 75-83.

Rustemeyer, J., \& Gregersen, J. (2012). Quality of life in orthognathic surgery patients. Post-surgical improvements in aesthetics and self-confidence. Journal of CranioMaxillo-Facial Surgery, 40(5), 400-404. doi: http://dx.doi. org/10.1016/j.jcms.2011.07.009

Sadek, H., \& Salem, G. (2007). Psychological aspects of orthognathic surgery and its effect on quality of life in Egyptian patients. La Revue de Santé de la Méditerranée Orientale, 13(1), 150-159.

Seidl, E. M. F., \& Zannon, C. M. L. C. (2004). Qualidade de vida e saúde: aspectos conceituais e metodológicos. Cad. Saúde Pública, 20(2), 580-588. Doi: http://dx.doi.org/10.1590/ S0102-311X2004000200027

Shaw, W. C. (1981). The influence of children's dentofacial appearance on their social attractiveness as judged by peers and lay adults. Am. J. Orthod., 79, 399-415. doi: http:// dx.doi.org/10.1016/0002-9416(81)90382-1

The Whoqol Group. (1995). The World Health Organization quality of life assessment (WHOQOL): Position paper from the World Health Organization. Soc. Sci. Med., 41(10), $1403-1410$.

The Whoqol Group. (1998). Development of the World Health Organization WHOQOL-bref. Quality of life assessment. Psychol. Med., 28, 551-558.

Turvey, T. A., Phillips, C., Zaytoun, H. S., \& Proffit, W. R. (1988). Simultaneous superior repositioning of the maxilla and mandibular advancement. Am. J. Orthod. Dentofac. Orthop., 94, 372-83. doi: http://dx.doi.org/10.1016/08895406(88)90126-6

Vieira, L. C. (2010). Tradução, adaptação cultural e validação de face e conteúdo do psychossocial impact of dental aesthetics questionnaire para uso no Brasil. Dissertação de mestrado. Universidade Federal de São Paulo, SP.

Zhou, Y. H., Hagg, U., \& Rabie, A. B. M. (2001). Patient satisfaction following orthognathic surgical correction of skeletal class III malocclusion. Int. J. Adult Orthod. Ortognath. Surg., 16(2), 99-107.

Westermarck, A., Shayeghi, F., \& Thor, A. (2001). Temporomandibular dysfunction in 1516 patients before and after orthognathic surgery. Int. J. Adult Orthod. Orthognath Surg., 16(2), 145-151. 